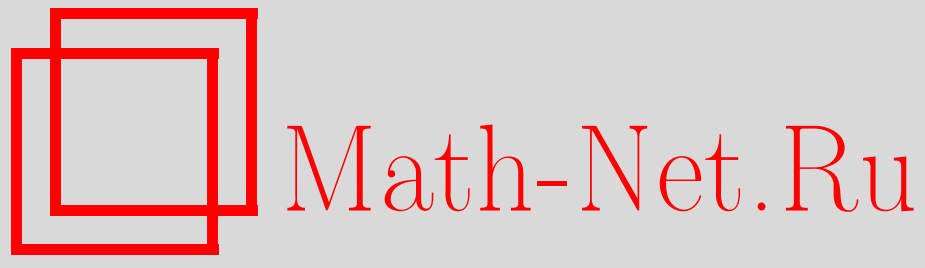

В. И. Арнольд, Самые длинные кривые данной степени и квазикристаллическая теорема Харнака в псевдопериодической топологии, Функи. анализ и его прил., 2002, том 36, выпуск 3, 1-8

DOI: https://doi.org/10.4213/faa199

Использование Общероссийского математического портала Math-Net.Ru подразумевает, что вы прочитали и согласны с пользовательским соглашением

http://www . mathnet.ru/rus/agreement

Параметры загрузки:

IP: 52.90 .164 .192

26 апреля 2023 г., 07:31:03

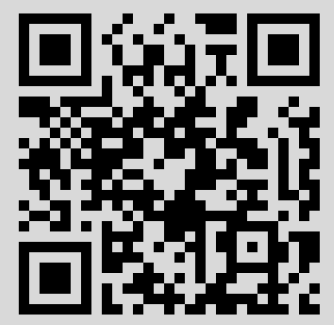




\title{
Самые длинные кривые данной степени и квазикристаллическая теорема Харнака в псевдопериодической топологии*
}

\author{
(c) 2002. В. И. Арнольд
}

\section{§1. Максимальные длины тригонометрических и сферических кривых}

Тригонометрическая кривая «степени $n$ »

$$
\{f(x, y)=0\}, \quad \text { где } f=\sum\left[a_{p, q} \cos (p x+q y)+b_{p, q} \sin (p x+q y)\right],
$$

задается вещественным тригонометрическим многочленом $f$ степени $n$ по каждой переменной $(|p| \leqslant n,|q| \leqslant n)$ на торе $\{(x \bmod (2 \pi), y \bmod (2 \pi))\}$. Снабдим тор стандартной метрикой $(d x)^{2}+(d y)^{2}$.

Оказывается, длина кривой (1) ограничена сверху постоянной, не зависящей от коэффициентов тригонометрического многочлена $f$ :

Теорема 1. Длина всякой кривой (1) степени $n$ не превосходит числа $C n$, где $C=8 \pi$.

ЗАМЕЧАНИЕ. Значение $8 \pi$ достигается для набора $4 n$ окружностей,

$$
f=\sin (n x) \sin (n y) .
$$

Теорема 1 утверждает, что это - самая длинная среди тригонометрических кривых cmenени $n$.

ДОКАЗАТЕЛЬСТВО ТЕОРЕМЫ 1. Пересечение кривой (1) с почти каждой из окружностей $x=$ const или $y=$ const состоит из $2 n$ точек. Поэтому длина проекции кривой на окружность $\{x=0\}$ вдоль оси $y$ (или на окружность $\{y=0\}$ вдоль оси $x$ ) не превосходит $2 \pi \cdot 2 n$ (считая длину проекции любого элемента кривой положительной, независимо от ориентации).

Поскольку длина гипотенузы не превосходит суммы длин катетов, длина всей кривой (1) не превосходит числа $2(2 \pi \cdot 2 n)=C n$, что и доказывает теорему.

ЗАмечАниЕ. Так же доказывается ограниченность сверху $k$-мерного объема $k$-мерного тригонометрического многообразия степени $n$ в торе размерности $m$ (объем ограничен величиной $C n$, где постоянная $C$ зависит только от размерностей $k$ и $m$, но не зависит от степеней $n$ и от коэффициентов тригонометрических многочленов $f$, задающих изучаемое многообразие $\{f=0\}$ ).

Рассмотрим теперь единичную сферу в трехмерном евклидовом пространстве с координатами $(x, y, z)$. На сфере рассмотрим алгебраическую кривую $\{f(x, y, z)=0\}$, где $f-$ однородный многочлен степени $d$. Степень $n$ этой сферической кривой равна $2 d$.

Оказывается, длина такой сферической алгебраической кривой степени $n$ ограничена сверху постоянной, не зависящей от коэффициентов многочлена $f$.

\footnotetext{
*Частично поддержано РФФИ, грант 02-01-00655.
} 
Теорема 2. Длина всякой сферической алгебраической кривой степени $n$ не превосходит числа $C$, где $C=\pi$ (для сферы радиуса единица).

ДокАЗАТЕЛЬство. Число точек пересечения нашей кривой с окружностью большого круга сферы не превосходит $n$ (по теореме Безу).

Среднее число точек пересечения со всеми окружностями больших кругов, вычисленное для любого бесконечно малого отрезка кривой, пропорционально длине этого отрезка, с не зависящим от отрезка коэффициентом пропорциональности (принцип «задачи Бюффона об иголке» из теории вероятностей, см., например, [7]).

Поэтому среднее число точек пересечения со всеми окружностями больших кругов, вычисленное для любой гладкой сферической кривой, пропорционально длине кривой, с тем же, что для бесконечно малого отрезка, коэффициентом пропорциональности.

Этот коэффициент равен $1 / \pi$, так как для окружности одного из больших кругов число точек ее пересечения с почти любой окружностью большого круга равно 2, а длина равна $2 \pi$.

Выше доказано уже, что для кривой степени $n$ среднее число точек ее пересечения с окружностями больших кругов не превосходит $n$. Значит, длина этой кривой не превосходит $\pi n$.

ЗАмЕЧАНИЕ 1 . Кривая степени $n=2 m$, состоящая из $m$ окружностей больших кругов, имеет длину $2 \pi m=\pi n$. Теорема 2 утверждает, что это - самая длинная среди всех сферических кривых такой же степени.

Замечание 2 . Величина $k$-мерного объема $k$-мерного подмногообразия степени $n$ в сфере размерности $m$ оценивается сверху (совершенно по тем же соображениям, что для кривых) величиной $C n$, где постоянная $C$ зависит только от размерностей $k$ и $m$ (но не зависит ни от степеней, ни от коэффициентов многочленов $f$, задающих изучаемое многообразие $\{f=0\}$ ).

Замечание 3. Равенство в теореме 2 для алгебраических кривых достигается только в случае, когда кривая - объединение окружностей больших кругов.

Ибо будь на кривой точка ненулевой геодезической кривизны, кривая пересекала бы некоторые окружности больших кругов в меньшем, чем $n$, числе точек, так что среднее число точек пересечения было бы строго меньше $n$, а длина кривой - строго меньше, чем $\pi n$.

Но неалгебраические сферические кривые «топологической степени $n$ » (пересекающие окружность каждого большого круга не более, чем $n$, раз) могут иметь указанную максимальную длину, не будучи объединением окружностей больших кругов. Пример - четыре не продолжающих друг друга меридиана сферы.

Я не знаю структуры ни множества всех кривых топологической степени $n$, ни его подмножества кривых экстремальной длины.

ЗАмечАниЕ 4. Теоремы 1 и 2 можно сравнивать, вложив тор в трехмерную сферу пространства $\mathbb{C}^{2}$ :

$$
(x, y) \mapsto(z=\exp (i x), w=\exp (i y)) .
$$

Но нужно учитывать, что степень $n$ вещественного многообразия $\{f=0\}$ не совпадает со степенью $N$ алгебраического многообразия, заданного обращением в нуль вещественной и мнимой частей комплексной функции $f$ (а именно, $N=n^{2}$ ). 


\section{§2. Оценки средних топологических и геометрических характеристик псевдопериодических многообразий и функций через их многогранники Ньютона}

Псевдопериодическое многообразие в пространстве $\mathbb{R}^{m}$ определяется как пересечение $m$-периодического подмногообразия с аффинным подпространством (какой-либо размерности $k$ ) в $\mathbb{R}^{m}$. Здесь $m$-периодичность - это инвариантность относительно сдвигов на все целочисленные векторы (т. е. на векторы из подрешетки $\mathbb{Z}^{m}$ в $\mathbb{R}^{m}$ ). Указанное аффинное пространство, вообще говоря, иррационально, т.е. несоизмеримо с этой подрешеткой (ненулевые разности его векторов решетке не принадлежат).

Пример псевдопериодического многообразия - множество нулей функции $\cos t+\cos (\sqrt{2} t)$. Периодическое подмногообразие ${ }^{1)}$ здесь - это плоская кривая, заданная уравнением $\cos (2 \pi x)+\cos (2 \pi y)=0$ в $\mathbb{R}^{2}(m=2)$. Аффинное одномерное подмногообразие - это прямая $\{2 \pi x=t, 2 \pi y=\sqrt{2} t\}$.

Псевдопериодические функции - это суммы линейных функций и периодических функций.

Эргодические средние (на единицу $k$-мерного объема) определяются для таких геометрических и топологических характеристик псевдопериодических объектов, как интегралы, объемы, длины, числа Бетти, эйлеровы характеристики, сигнатуры и иные характеристические числа, числа Морса критических точек разных индексов и т. п.

Для определения среднего выбирается точка (например, $O$ ) и затем изучаемый объект (например, функция, или ее множество уровня, или ее многообразие меньших значений, или ее множество критических точек, или иное псевдопериодическое многообразие) рассматривается внутри шара большого радиуса $R$ в $\mathbb{R}^{m}$ с центром в точке $O$.

Топологические или геометрические характеристики изучаемого объекта (числа Бетти, числа Морса, интегралы, длины, объемы и т.п.) вычисляются для получившегося в пересечении с шаром ограниченного объекта.

Полученная при вычислении величина делится на объем шара (иногда в $\mathbb{R}^{m}$, а иногда и в $k$-мерном изучаемом аффинном подпространстве), а затем радиус шара, $R$, устремляется к бесконечности.

Предел отношения при стремящемся к бесконечности радиусе $R$ называется эргодическим средним изучаемой характеристики (на единицу объема).

Этот предел существует почти всегда (при почти всех центрах $O$ в смысле меры Лебега), а иногда даже и всегда (как я сформулировал в [1] и как С. М. Гусейн-Заде доказал в $[2,3])$.

Ниже обсуждается поставленный в [1] и в [6] вопрос об оценке сверху эргодических средних на единицу объема от характеристик псевдопериодических объектов, полученных из периодического его сечением $k$-мерным аффинным подпространством. Оценку сверху доставляет степень (или многогранник Ньютона) указанного периодического объекта. Константы в оценках зависят от того, рассматривается ли $2 \pi$-периодичность, как в $\$ 1$, или 1 -периодичность, как в $\$ 2$.

1) Переход от решетки $(2 \pi \mathbb{Z})^{m}$ из $§ 1$ к употребляемой здесь решетке $\mathbb{Z}^{m}$ меняет лишь постоянные $C$ в оценках и масштаб длин. 
ЗАмЕчАНИЕ. В некоторых случаях (например, при изучении длины линии уровня псевдопериодической функции $f$ от двух переменных $x$ и $y$ ) включение вопроса в приведенную выше схему требует дополнительных усилий (в указанном примере нужно перейти от функции $f$, не являющейся ограничением на иррациональную плоскость периодической функции большего числа переменных, к функции

$$
\frac{\partial f / \partial x}{\sqrt{(\partial f / \partial x)^{2}+(\partial f / \partial y)^{2}}}
$$

уже являющейся таким ограничением (как является и $\partial f / \partial x)$ ).

Рассмотрим (тригонометрическую) кривую длины $L$ на стандартном двумерном торе $T^{2}=\mathbb{R}^{2} / \mathbb{Z}^{2}$. Соответствующую периодическую кривую на плоскости $\mathbb{R}^{2}$ обозначим через $M$.

Теорема 3. Эргодическое среднее (на единииу длинь секущей прямой) от числа точек пересечения кривой $M$ с иррациональной аффинной прямой ограничено сверху величнной $C L$ (где постоянная $C$ не зависит от выбора прямой).

ДокАЗАТЕЛЬСтво. Ориентировав прямую, сопоставим каждой точке кривой $M$ отрезок длины 1 , начинающийся в этой точке и направленный в определенную ориентацией сторону, параллельно прямой.

Сложим характеристические функции всех построенных параллельных отрезков (характеристическая функция равна 1 в точках отрезка и 0 вне его). Сумма, $f,-$ периодическая функция на плоскости, определенная всюду или по меньшей мере почти всюду и уж заведомо интегрируемая по мере Лебега (так как число точек пересечения любого отрезка прямой с кривой $M$ ограничено сверху зависящей лишь от длины отрезка постоянной в силу полуалгебраичности исходной тригонометрической кривой на торе).

Мы будем считать периодическую функцию $f$ результатом поднятия на накрывающую тор плоскость неотрицательной функции $g$, заданной на торе.

Интеграл от функции $g$ (как от плотности распределения $g d S$ ) по всему тору ограничен сверху постоянной, не зависящей от направления секущей прямой и пропорциональной длине $L$ исходной тригонометрической кривой на торе.

Действительно, распределение $g d S$ можно получить, интегрируя, как по параметру, по точке исходной кривой распределение наших параллельных отрезков, перенесенное с плоскости на тор проектированием.

А именно, фиксированной точке исходной кривой отвечает на плоскости равномерное распределение единичной массы вдоль выходящего из этой точки по направлению секущей прямой отрезка.

При проектировании плоскости на тор это распределение переходит в свой прямой образ, т. е. если в точку на торе проектируется несколько отрезков плоскости, порожденных разными точками исходной периодической кривой, то принесенные ими распределения суммируются.

Число точек пересечения интервала длины $T$ секущей прямой с периодической кривой $M$ на плоскости не сильно отличается от интеграла от построенной нами на плоскости функции $f$ вдоль этого интервала, даже когда он очень длинен.

Действительно, каждый из наших параллельных отрезков длины 1 , который целиком умещается в рассматриваемый интервал длины $T$, дает одинаковые вклады 1 и в интеграл от $f$, и в число точек пересечения. 
Число же отрезков, участвующих в построении функции $f$, которые пересекаются с концами нашего интервала длины $T$, ограничено сверху постоянной (которая даже не зависит от направления прямой и вычисляется по степени тригонометрического многочлена, задающего исходную кривую на торе).

Это же число оценивает и число тех точек пересечения, которые не сопровождаются отрезком, целиком попадающим в наш интервал (начинающимся в точке пересечения).

Поэтому эргодические средние (на единицу длины секущей прямой) от числа точек пересечения с $M$ и от ограничения на прямую функции $f$, в пределе $T \rightarrow \infty$, одинаковы. Значит (по эргодической теореме для иррациональной секущей прямой) временно́е среднее числа точек пересечения ограничено той же постоянной, что и пространственное среднее от функции $g$ по всему тору.

Последнее, в свою очередь, ограничено сверху, как мы доказали выше, величиной $C L$. Это доказывает теорему 3.

Следствие. Среднее (на единицу длины секущей прямой) от числа нулей псевдопериодической функиии, являющейся ограничением на иррациональную прямую тригонометрического многочлена степени $n$, ограничено сверху величиной $C$ (где постоянная $C$ не зависит от коэффициентов многочлена).

ДокАЗАТЕЛЬСтво. Длина $L$ линии нулей тригонометрического многочлена степени $n$ ограничена сверху величиной, пропорциональной $n$ (по теореме 1 ), а среднее число нулей $K$ псевдопериодического ограничения - величиной, пропорциональной длине $L$ (по теореме 3 ). Итак,

$$
K \leqslant C_{1} L \leqslant C_{1} C_{2} n,
$$

что и утверждалось.

ЗАМЕЧАНИЕ 1. Сечение периодического подмногообразия размерности $d$ в пространстве размерности $m$ аффинным подпространством размерности $k$ имеет размерность $s=k+d-m$. Если $s \geqslant 0$, то эргодическое среднее (на единицу $k$-мерного объема) от величины $s$-мерного (неориентированного) объема псевдопериодического сечения оценивается сверху величиной $C n$, где $n-$ степень периодического многообразия.

Доказывается это так же, как теорема 3 и ее следствие, только вместо отдельных точек (пересечения секущей прямой с заданной периодической кривой) нужно рассматривать подмногообразия (размерности $s=k+d-r$ ) пересечения $k$-мерного секущего пространства с $d$-мерным периодическим подмногообразием B $\mathbb{R}^{m}$.

Место числа этих точек пересечения занимает $s$-мерный объем многообразияпересечения. Отрезки длины 1 (в определении функций $f$ и $g$ ) нужно заменить шарами (или кубами) радиуса единица.

Теорема о совпадении временно́го среднего с пространственным вдоль иррациональной обмотки тора (относящаяся к эргодической теории динамических систем) заменяется аналогичной (и доказываемой таким же анализом ряда Фурье) теоремой об усреднении вдоль иррационального $k$-мерного слоения на $m$-мерном торе.

Трюк с построением функции $f$ (из характеристических функций отрезков) был придуман Г. Вейлем для задачи о среднем движении перигелия Земли $[5,4]$, где этот прием позволил ему регуляризовать расходящийся интеграл. 
ЗАмечАниЕ 2. Теоремы об оценках сверху эргодических средних от других топологических и геометрических характеристик псевдопериодических и квазикристаллических объектов доказываются аналогичными теореме 3 приемами.

Рассмотрим, например, критические точки той псевдопериодической функции, в которую превращается тригонометрический многочлен $f(x, y, z)$ (степени $n$ по каждой переменной) при его ограничении на иррациональную плоскость, $a x+$ $b y+z=0$.

В критической точке ограничения градиент периодической функции $f$ пропорционален вектору $(a, b, c)$, так что критические точки удовлетворяют обоим тригонометрическим уравнениям

$$
b \frac{\partial f}{\partial x}=a \frac{\partial f}{\partial y}, \quad c \frac{\partial f}{\partial y}=b \frac{\partial f}{\partial z} .
$$

Каждое из этих уравнений имеет степень $n$, так что заданная этими уравнениями кривая в трехмерном торе - тригонометрическая, степени порядка $n^{2}$.

Длина такой кривой ограничена сверху величиной $C n^{2}$, не зависящей от коэффициентов тригонометрического многочлена $f$. Рассуждая, как в теореме 3 и в доказанном следствии, мы получим для эргодического среднего (на единицу площади плоскости аргументов) от числа критических точек изучаемой псевдопериодической функции оценку сверху величиной порядка $n^{2}$ (для функций от $k$ переменных и среднего на единицу $k$-мерного объема получилась бы оценка сверху среднего числа критических точек величиной порядка $n^{k}$ ).

Примеры, показывающие неулучшаемость этих оценок, очевидны (например, $f=\cos (n x)+\cos (n y)+\cos (n z))$.

\section{§3. Псевдопериодическая теорема типа Харнака}

Рассмотрим псевдопериодическую кривую степени $n,\{f(x, y, z)=0\}$, на иррациональной плоскости $\{a x+b y+c z=0\}$. Исследуем число компонент связности пересечения этой кривой с шаром большого радиуса $R$.

Теорема 4. Число таких компонент не превосходит величины $\mathrm{Cn}^{2} R^{2}$.

ДокАЗАТЕльство. Пусть компонента не пересекает границу рассматриваемого круга радиуса $R$ на плоскости. В области, ограниченной этой компонентой, не доходя до лежащих в этой области других компонент кривой, если таковые имеются, псевдопериодическая функция $f$ имеет критическую точку. Эти точки у разных компонент разные, поэтому число таких компонент не превосходит числа $C_{1} n^{2} R^{2}$, оценивающего сверху число критических точек в круге по теореме 3 и ее следствию.

Чтобы оценить сверху число компонент, пересекающих граничную окружность круга радиуса $R$, аппроксимируем эту окружность ломаной из отрезков постоянства координат $x$ и $y$. Число точек пересечения кривой $\{f=0\}$ с такой ломаной оценивается сверху величиной произведения степени $n$ на длину ломаной.

Те же компоненты связности кривой $\{f=0\}$, которые пересекают граничную окружность, но не пересекают аппроксимирующую ломаную, содержат точки обращения вспять, где $f=0$ и либо $\partial f / \partial x=0$, либо $\partial f / \partial y=0$. Число этих компонент тоже оценивается величиной порядка $n^{2} R^{2}$ по теореме 3 и ее следствию. 
Складывая оценки чисел компонент связности всех трех типов, мы получим оценку сверху для их общего числа вида $C_{1} n^{2} R^{2}+C_{2} n R+C_{3} n^{2} R^{2}$, что и доказывает теорему 4.

\section{§4. Псевдопериодическая теорема типа Штурма-Гурвица}

Теорема Штурма-Гурвица (см., например, [6]) оценивает топологическую сложность тригонометрического многочлена не сверху, как теоремы Безу и Харнака, а снизу, в предположении отсутствия в многочлене не членов высокой, а, наоборот, членов низкой степени.

Я укажу здесь простейшие оценки длины тригонометрических кривых снизу. Вернемся к обозначениям формулы (1).

Теорема 5. Предположим, ито степень каждого илена тригонометрического многочлена (или ряда Фурье) f по х не меньше $n$ (т. е. что в представлении (1) всюду $|p| \geqslant n)$.

тогда длина кривой $\{f(x, y)=0\}$ не меньше, чем $4 \pi n$ (каковая длина достигается для $f=\cos (n x))$.

ДоКАЗАТЕЛЬСтвО. При фиксированном значении $y$ многочлен $f(\cdot, y)$ имеет не менее $2 n$ корней $x$ (по теореме Штурма-Гурвица). Поэтому кривая $\{f=0\}$ при проектировании вдоль оси $x$ на ось $y$ покрывает эту окружность длины $2 \pi$ не менее $2 n$ раз, что и доказывает теорему.

ЗАмЕчАниЕ. Для вещественного тригонометрического многочлена $f$ от $m$ переменных такое же рассуждение дало бы оценку $(m-1)$-мерного объема гиперповерхности $\{f=0\}$ снизу величиной $(2 \pi)^{m-1} 2 n$ (в теореме 5 и здесь, как и в (1), метрика тора определяется суммой квадратов дифференциалов угловых координат).

Обобщение на случай подмногообразий, коразмерность которых больше единицы (например, на случай системы двух уравнений на двумерном торе), к сожалению, неизвестно.

Штурм связывал одно из доказательств своей теоремы с выражением индекса комплексного векторного поля на окружности $|z|=1$ через число нулей на этой окружности вещественной части поля. Эта связь в многомерном случае также не исследована. В $n$-мерном случае окружность заменяется сферой $S^{2 n-1}$ в комплексном пространстве $\mathbb{C}^{n}$, а вещественная часть комплексного векторного поля - лагранжевой проекцией ограничения голоморфного на сфере симплектического пространства векторного поля на лежащий на сфере лагранжев тор $\left|z_{k}\right|=r_{k}$.

СледствиЕ. Эргодическое среднее (на единицу длинь секущей прямой) от числа нулей псевдопериодического ограничения многочлена теоремь 5 на иррацииональную прямую оценивается снизу величиной $\mathrm{Cn}$.

Так же оценивается снизу и среднее от объема в ситуации предыдущего замечания. Все это выводится из теоремы 5 так же, как выведено выше следствие теоремы 3 из теоремы 1.

ЗАмЕЧАНИЕ 1. Тем же методом оценивается снизу и длина псевдопериодической кривой $\{f(x, y, z)=0\}$ на иррациональной плоскости $\{a x+b y+c z=0\}$, если степень $|p|$ каждого члена многочлена $f$ не меньше $n$. Длина той части кривой, которая заключена в шаре радиуса $R$, растет с $R$ в этом случае не медленнее, чем $C R n$. Здесь также кривую на плоскости можно заменить гиперповерхностью в пространстве. 
ЗАмечание 2. Классическая теорема Харнака оценивает сверху число компонент алгебраической вещественной кривой через род ее римановой поверхности. В многомерном случае теорема Смита оценивает сверху сумму чисел Бетти вещественного алгебраического многообразия суммой чисел Бетти множества его комплексных точек.

Подобная связь между вещественными псевдопериодическими кривыми (или многообразиями) и поверхностями (множествами) их комплексных точек тоже должна иметь место, но отсутствует в известной мне литературе.

ЗАмечАниЕ 3. Длина $L$ кривой на торе (соответственно объем подмногообразия) доставляет оценку снизу для величины усредненного по секущим прямым (подпространствам) всех направлений эргодического среднего от числа (объема) псевдопериодических пересечений аффинного подпространства накрывающего пространства с периодической накрывающей исходной кривой (исходного подмногообразия).

Отсюда вытекает существование таких длинных сечений (сечений большого эргодического среднего объема) при надлежащем выборе аффинного подпространства. Интересно узнать, насколько редки более короткие сечения.

\section{ЛИТЕРАТУРА}

1. Arnold V. I. Remarks on quasicrystallic symmetries. Phys. D, 33, 21-25 (1988).

2. Gusein-Zade S. M. On the topology of quasiperiodic functions. In: Pseudoperiodic Topology (V. Arnold, M. Kontsevich, and A. Zorich, eds.). Amer. Math. Soc. Transl., Ser. 2, Vol. 197, 1999, pp. 1-7.

3. Гусейн-Заде С. М. Число критических точек квазипериодического потенциала. Функц. анализ и его прил., 23, вып. 2, 55-56 (1989).

4. Weyl H. Mean Motion I. Amer. J. Math., 60, 889-896 (1938).

5. Weyl H. Mean Motion II. Amer. J. Math., 61, 143-148 (1939).

6. Арнольд В. И. и др. Задачи Арнольда. Фазис, М., 2000.

7. Арнольд В. И. Вариация кривой. Матем. просвещение, вып. 2, М., 1957, с. 241-245.

Математический институт им. В. А. Стеклова, CEREMADE, Universitè Paris-Dauphine 